\title{
Reconstruction nailing for ipsilateral femoral neck and shaft fractures
}

\author{
Alexandros Tsarouhas • Michael E. Hantes • \\ Theohilos Karachalios • Konstantinos Bargiotas • \\ Konstantinos N. Malizos
}

Received: 10 July 2010/Accepted: 11 July 2011/Published online: 21 July 2011

(C) The Author(s) 2011. This article is published with open access at Springerlink.com

\begin{abstract}
The surgical management of ipsilateral fractures of the femoral neck and shaft presents a difficult and challenging problem for the orthopaedic surgeon. The purpose of the present study was to report the mid-term results and complications in a series of patients who sustained ipsilateral femoral neck and shaft fractures and treated in our trauma department with a single reconstruction nail for both fractures. Eleven patients were included in the study with an average age of 46.4 years. The mean follow-up was 47 months (range, 15-75 months). There were no cases of a missed diagnosis at initial presentation. The mean time to union was 4.5 months for the neck fracture and 8.2 months for the shaft. There were no cases of avascular necrosis of the femoral head or non-union of the neck fracture. The mean Harris Hip Score was $(85 \pm 4.3)$. Complications included two cases of shaft fracture non-union and one case of peroneal nerve palsy. Heterotopic ossification at the tip of the greater trochanter was evident in two cases without causing any functional deficit. The current study suggests that reconstruction nailing produces satisfactory clinical and functional results in the mid-term. The complications involved only the femoral shaft fracture and were successfully treated with a single operative procedure.
\end{abstract}

A. Tsarouhas · M. E. Hantes $(\bowtie) \cdot$ T. Karachalios ·

K. Bargiotas $\cdot$ K. N. Malizos

The Department of Orthopaedic Surgery,

University Hospital of Larissa,

School of Health Sciences,

University of Thessalia, Mezourlo,

41110 Larissa, Greece

e-mail: hantesmi@otenet.gr
Keywords Femoral shaft fractures - Ipsilateral hip · Reconstruction nail

\section{Introduction}

Ipsilateral femoral neck and shaft fractures commonly occur in young adults and are the result of high-energy injuries such as road traffic accidents or a fall from a height. Although relatively rare, the incidence ranges between 2.5 and $9 \%$ of all femoral shaft fractures $[1,21]$ but seems to be rising [11].

Surgical management of ipsilateral fractures of the femoral neck and shaft is a difficult and challenging problem for the orthopaedic surgeon. The timing of fracture fixation, implant selection and sequence of fixation must be considered. Numerous implants have been recommended for the fixation of these injuries, but no consensus has been reached as to the optimal treatment strategy. The selected implant should facilitate anatomic fracture reduction and stable fixation, both of which have been shown to favour early mobilization, high union rates and reduced complication rates $[7,17]$. The use of a single cephalomedullary nail has been advocated based on the ease of application and decreased surgical time and blood loss $[10,11]$. Other reports suggest that internal fixation of the fractures with two separate implants provides better results in terms of fracture reduction and complication rates $[12,13]$.

The available data on the success of each fixation device are derived from case series mainly in which a limited number of patients were treated in different trauma centres and with varying techniques. The purpose of our study was to report the mid-term results in a series of patients treated in our trauma department with a single reconstruction nail for both fractures. 


\section{Patients and methods}

Between January 2004 and December 2008, eleven male patients with an average age of 46.4 years (range, 18-75 years) were diagnosed with ipsilateral femoral neck and shaft fractures and treated in our department. All resulted from motor vehicle accidents. The right side was affected in 7 cases. There was no life-threatening trauma, but five patients sustained other orthopaedic-related injuries: two patients had ipsilateral patella fractures and one also sustained a contralateral ulna fracture; another patient had an ipsilateral ischiopubic and a contralateral radius fracture; a contralateral pilon fracture and a large rotator cuff tear were diagnosed in two other patients (Table 1).

Injury radiographs were assessed to classify the fractures. Plain radiographs of the pelvis as well as anteroposterior and lateral views of the femur and knee were routinely performed in the emergency room in case of suspected femoral fractures as part of a standard trauma assessment protocol. Eight of the femoral neck fractures were basicervical while the remaining three were midcervical. Nine of the neck fractures were non-displaced or minimally displaced. Femoral shaft fractures were classified according to Winquist and Hansen [22]. There were three type 0 , four type I, two type II and two type III fractures. There were 6 middle third and 5 distal third fractures. Two of the femoral shaft fractures were open (Gustilo-Anderson type II) (Table 1).

Surgical management of the fractures was performed after initial assessment and resuscitation. The patients were placed supine on a fracture table. Closed reduction was achieved in all cases under traction with slight abduction and internal rotation. In cases of open fractures, wound debridement and closure preceded the femoral shaft nailing.
The reconstruction nail was inserted after reduction of the shaft fracture. Careful X-ray monitoring during reaming and nail insertion was carried out to prevent displacement of the neck fracture. Bone grafting was not performed even in cases of severe comminution. Following the insertion of the nail, accurate reduction of the femoral neck fracture was checked for again prior to proximal screw insertion. In all but two cases, a single proximal 10.5-mm interlocking screw at $125^{\circ}$ insertion angle (Long Gamma nail, Stryker) was used for the fixation of the neck fracture. In the remaining two patients, a Russel-Taylor Delta reconstruction nail (Smith \& Nephew Richards Inc. Memphis, Tennessee USA) with two proximal screws was inserted. An attempt was made to place the neck screw in the centre of the femoral head in both frontal and lateral views. The femoral neck fracture was provisionally stabilized with Steinmann pins to prevent displacement or rotational malalignment of during drilling. Distal locking was performed with either one or two screws. Fluoroscopy was routinely used to confirm correct placement of proximal and distal locking screws in orthogonal views. Concomitant fractures were addressed; a pelvic external fixator was applied for the patient with the ischiopubic fracture and internal fixation performed for the ulna and patella fractures.

Early mobilization was encouraged post-operatively but weight-bearing restricted for the first 8 weeks. Partial weight bearing was then allowed based on radiographic signs of callus formation. Passive knee range of motion and isometric quadriceps-strengthening exercises were started from the second post-operative day.

Radiographs at injury, post-surgery and during followup were reviewed to assess the quality of fracture reduction and time to union. The follow-up radiographs were repeated on a monthly basis until union of both fractures was

Table 1 Patient profile

\begin{tabular}{|c|c|c|c|c|c|c|c|}
\hline $\begin{array}{l}\text { Patient } \\
\text { number }\end{array}$ & Age & Gender & Side & Femoral neck fracture & $\begin{array}{l}\text { Femoral shaft } \\
\text { fracture }\end{array}$ & $\begin{array}{l}\text { Open/closed } \\
\text { fracture }\end{array}$ & Associated injuries \\
\hline 1 & 18 & M & $\mathrm{L}$ & Mid-cervical, non-displaced & Middle third, WH-I & Open, G-A II & - \\
\hline 2 & 75 & M & $\mathrm{R}$ & Basicervical, non-displaced & Middle third, WH-I & Closed & - \\
\hline 3 & 52 & M & $\mathrm{L}$ & Basicervical, displaced & Middle third, WH-I & Closed & Pillon \# \\
\hline 4 & 39 & M & $\mathrm{L}$ & Basicervical, non-displaced & Middle third, WH-II & Closed & - \\
\hline 5 & 52 & M & $\mathrm{R}$ & Basicervical, minimally displaced & Distal third, WH-I & Open, G-A II & Rotator cuff tear \\
\hline 6 & 32 & M & $\mathrm{R}$ & Basicervical, non-displaced & Distal third, WH-0 & Closed & $\begin{array}{c}\text { Ulnar \& patellar } \\
\text { \# (ipsilateral) }\end{array}$ \\
\hline 7 & 55 & M & $\mathrm{L}$ & Basicervical, non-displaced & Middle third, WH-0 & Closed & Ischiopubic \& radial \# \\
\hline 8 & 42 & M & $\mathrm{R}$ & Mid-cervical, non-displaced & Middle third, WH-III & Closed & - \\
\hline 9 & 50 & M & $\mathrm{R}$ & Basicervical, non-displaced & Distal third, WH-II & Closed & Patellar \# \\
\hline 10 & 40 & M & $\mathrm{R}$ & Basicervical, displaced & Distal third, WH-O & Closed & - \\
\hline 11 & 52 & M & $\mathrm{R}$ & Mid-cervical, non-displaced & Distal third, WH-III & Closed & _- \\
\hline
\end{tabular}

$M$ male, $L$ left, $R$ right, \# fracture, $W H$ Winquist and Hansen, $G$ - $A$ Gustillo-Anderson 
evident. Fracture union was defined using clinical (absence of pain during weight bearing) and radiographic (bringing callus across 3 out of 4 cortices of the fracture) criteria [8]. The quality of fracture reduction was graded according to the criteria defined by Haidukewych et al. as excellent $\left(<2 \mathrm{~mm}\right.$ of displacement and $<5^{\circ}$ of angulation in any plane), good $\left(2-5 \mathrm{~mm}\right.$ of displacement and/or $5^{\circ}-10^{\circ}$ of angulation), fair ( $>5-10 \mathrm{~mm}$ of displacement and/or $>10^{\circ}-$ $20^{\circ}$ of angulation) or poor ( $>10 \mathrm{~mm}$ of displacement and/or $>20^{\circ}$ of angulation) [7]. Complications and their management were also noted. The functional results, including range of motion for the hip and knee joints as well as the Harris Hip Score (HHS), were assessed at the time of final follow-up.

\section{Results}

The mean follow-up was 47 months (range, 15-75 months) (Table 2). There were no cases of missed diagnosis at the time of initial presentation. The mean duration of surgery was $4.5 \mathrm{~h}$ (range, 3-6 h), which included the management of associated fractures. A single proximal screw was inserted in nine patients, while two screws were inserted in the remaining two. All the fractures were treated within 5-36 h of injury.

\section{Radiographic results}

At the final follow-up, reduction of the neck fracture was excellent in all 11 cases. The mean time to union for the neck fractures was 4.5 months (range, 4-6 months). There were no cases of avascular necrosis or non-union of the femoral neck. Apart from two cases of non-union, healing of the shaft fractures was evident, on average, 7.2 months after surgery (range, 6-9 months) (Table 2). Shaft alignment was normal in both coronal and sagittal planes except from one case of implant failure (distal screw breakage). The two patients with Winquist-Hansen type III shaft fractures showed a limb length discrepancy of approximately $1 \mathrm{~cm}$.

\section{Functional results}

The mean HHS was 85 (range, 78-92). Range of motion for the hip joint was normal in all the patients. Knee flexion averaged $120^{\circ}$ (range, 105-130). The two patients with the associated patellar fractures had the least knee flexion. Two patients, one with peroneal nerve palsy and another with a contralateral pilon fracture, reported slight limping. Two patients reported occasional pain that did not affect their everyday activities. All the patients returned to their preinjury activity level and occupation (Fig. 1).

\section{Complications}

There were two cases of shaft fracture non-union. In the first case, the fracture was originally fixed in distraction, which ultimately led to the breakage of the distal locking screws and valgus displacement of the shaft fracture at 5 months after surgery (Fig. 2). Revision surgery was performed to correct the deformity. The broken screws were replaced, and iliac autograft was used to induce union. A hypertrophic non-union of the shaft fracture occurred in the second case, which was treated by exchange nailing and bone grafting. In both cases, satisfactory union occurred 6 months after additional surgery (Fig. 3). There was one case of peroneal nerve palsy that presented immediately after surgery, presumed due to excessive traction during fracture reduction manoeuvres.
Table 2 Treatment and outcome data

HHS Harris Hip Score

\begin{tabular}{|c|c|c|c|c|c|c|c|}
\hline \multirow{2}{*}{ Patient no } & \multicolumn{2}{|c|}{ Locking screws } & \multirow{2}{*}{$\begin{array}{l}\text { Follow-up } \\
\text { (months) }\end{array}$} & \multicolumn{2}{|c|}{ Union time (months) } & \multirow[t]{2}{*}{ HHS } & \multirow[t]{2}{*}{ Complications } \\
\hline & Proximal & Distal & & Neck & Shaft & & \\
\hline 1 & 1 & 2 & 75 & 4 & 6 & 80 & Heterotopic ossification \\
\hline 2 & 1 & 1 & 33 & 4 & 7 & 84 & - \\
\hline 3 & 1 & 1 & 34 & 4 & 9 & 81 & - \\
\hline 4 & 1 & 2 & 67 & 5 & & 78 & $\begin{array}{l}\text { Hypertrophic shaft } \\
\text { non-union }\end{array}$ \\
\hline 5 & 1 & 2 & 50 & 4 & 8 & 85 & - \\
\hline 6 & 1 & 2 & 15 & 6 & & 92 & Distal screw breakage \\
\hline 7 & 2 & 2 & 55 & 5 & 7 & 89 & $\begin{array}{l}\text { Peroneal nerve palsy } \\
\text { heterotopic ossification }\end{array}$ \\
\hline 8 & 1 & 2 & 53 & 4 & 7 & 84 & - \\
\hline 9 & 2 & 2 & 41 & 4 & 8 & 90 & - \\
\hline 10 & 1 & 1 & 57 & 5 & 6 & 91 & - \\
\hline 11 & 1 & 2 & 37 & 6 & 9 & 81 & - \\
\hline
\end{tabular}



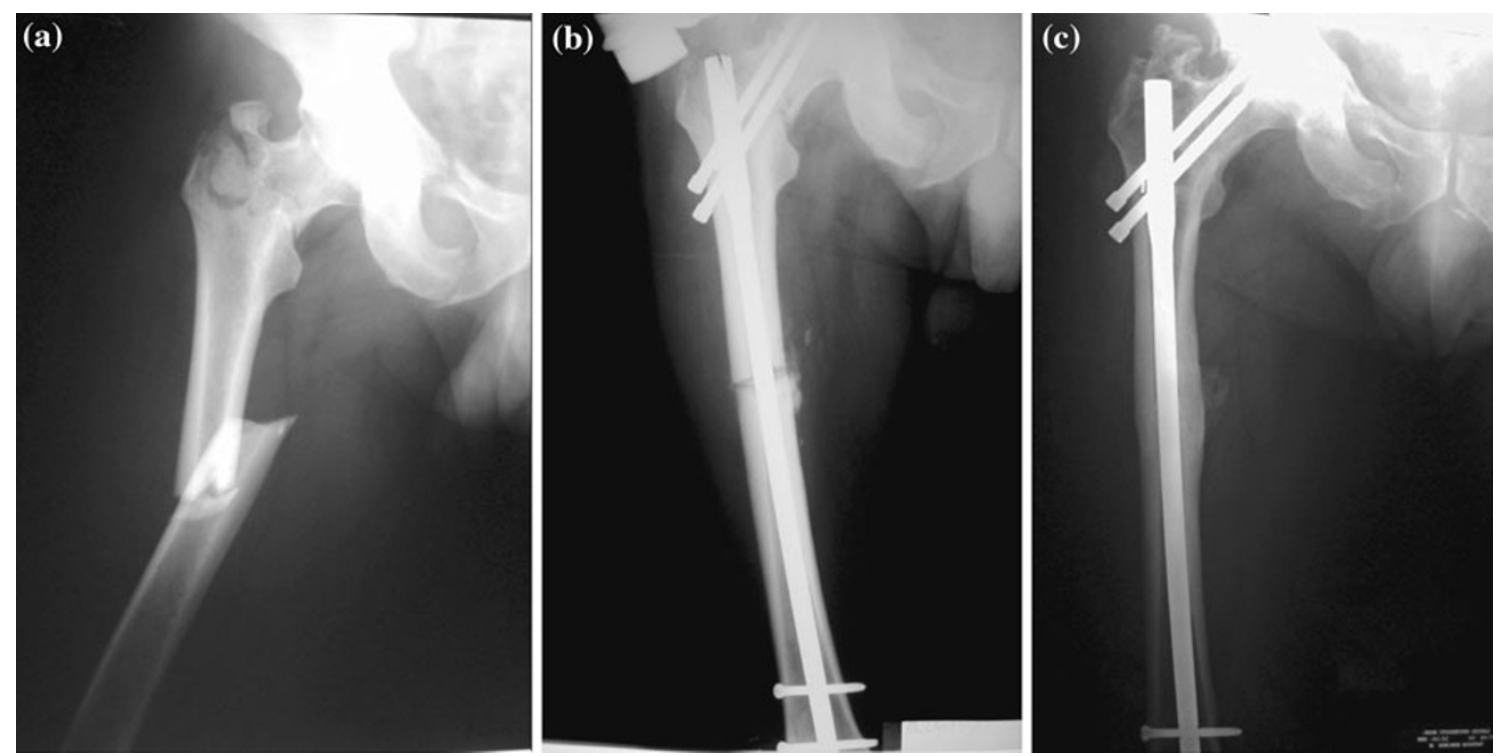

Fig. 1 Radiographs of ipsilateral femoral neck and shaft fracture (patient No 7): a pre-surgery, b after reconstruction nailing and $\mathbf{c}$ satisfactory union achieved after 8 months with evidence of heterotopic ossification at the tip of the great trochanter

At the final follow-up, the patient had grade 3 ankle extensor strength (Medical Research Council classification) and reported occasional difficulty to perform pre-injury everyday activities. Heterotopic ossification at the tip of the greater trochanter was radiographically evident in two cases without causing any functional deficit.

\section{Discussion}

In this, we report the mid-term results of treatment of a series of patients with ipsilateral femoral neck and shaft fractures, from a single-level one trauma centre, using a reconstruction nail for both fractures. Our results indicated that reconstruction nailing produces satisfactory functional and radiographic results. No cases of AVN were diagnosed, probably because of early surgical intervention and accurate fracture reduction, while the incidence of femoral shaft non-union was less than $20 \%$. Those cases of femoral shaft non-union were successfully resolved with one single additional episode of surgery.

In such injuries, there is an increased incidence of missed diagnosis of at least one of the two fractures during the initial assessment. The neck fracture is most commonly missed with an incidence ranging between 13 and 30\% [1, 14]. Cases of a delayed diagnosis of the femoral neck fracture have been reported even when computed tomography (CT) scans of the pelvis were performed [9]. Inadequate radiographic imaging, minimal displacement of the neck fracture and the presence of other orthopaedic or lifethreatening injuries are the commonest reasons for the neck fracture to be missed. Oblique or severely comminuted shaft fractures may also present with external rotation of the foot, potentially masking the clinical appearance of femoral neck fractures. Torneta et al. [18] recommend the use of a standard protocol, including a dedicated fine-cut CT scan, internal rotation plain radiographs of the hip as well as intra- and post-operative lateral hip radiographs. This showed a significant reduction of delay in diagnosis of these fractures. In this report, all the fractures were identified during initial assessment and resuscitation. This is attributable to the increasing awareness of this injury pattern and the routine use of pelvic and hip joint radiographs upon admission according to the ATLS ${ }^{\circledR}$ protocol.

Surgical management of ipsilateral femoral neck and shaft fractures has shown significantly better results compared with non-operative treatment [16]. Early surgical intervention allows for early mobilization and rehabilitation, thus reducing complications, morbidity and mortality, and improving overall outcomes [14]. A variety of fixation methods have been recommended to date; these include multiple flexible Ender nails with supplementary pinning, varying combinations of retrograde nailing or plate fixation for femoral shaft and dynamic hip screw or cannulated screw fixation for neck fractures, antegrade interlocking nailing with screw neck fixation and second-generation reconstruction (cephalomedullary) interlocking nailing. The optimal treatment protocol remains controversial.

The advantages of reconstruction nailing in the management of these fractures include minimal surgical trauma and blood loss, reduced operative time, single device positioning, biological fixation of the shaft fracture and a better aesthetic result [6]. When proximally and distally interlocked, this nail controls both length and rotation in 
Fig. 2 Radiographs of patient no 9: a pre-injury, $\mathbf{b}$ and c 5 months post-surgery. Valgus displacement had occurred as a result of distal screw breakage, d broken screws were replaced, and bone graft was inserted at the diaphyseal fracture site. IF hardware was also removed from the patella
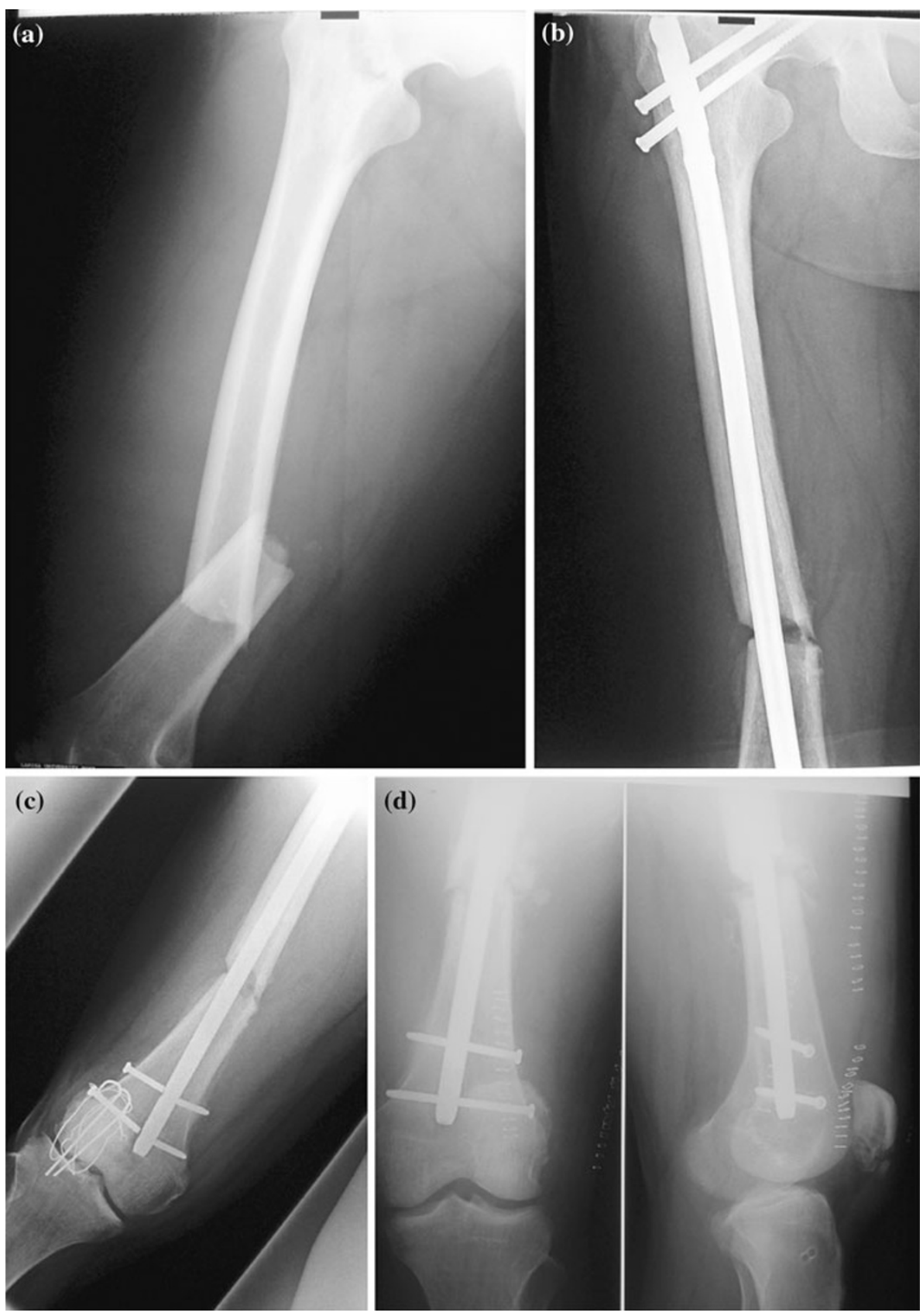

comminuted shaft fractures. Problems associated with alternative fixation methods, such as knee pain and stiffness from retrograde nailing or extensive dissection and stress shielding for plate fixation, are avoided with reconstruction nailing. The success rate of this procedure has been reported to range between 69 and 100\% [10, 11, 15]. However, several disadvantages have been reported. This procedure is technically demanding. Nail insertion may cause further displacement of an undisplaced or minimally displaced femoral neck fracture, which then may be difficult to reduce. Importantly, difficulties have been reported in obtaining rotational alignment of the fractures as well as in achieving correct positioning of the proximal interlocking screws [11]. Hardware removal and residual bone stock have also been shown to cause substantial difficulty during revision surgery [21].

There exists controversy currently regarding the use of a single or two separate devices for the fixation of this combination of fractures. In a recent comparative study, Bedi et al. [3] found that internal fixation of the neck fracture followed by retrograde nailing of the shaft fracture led to more accurate reduction and improved union rates compared with a single cephalomedullary device. On the other hand, in a retrospective comparison of four different 

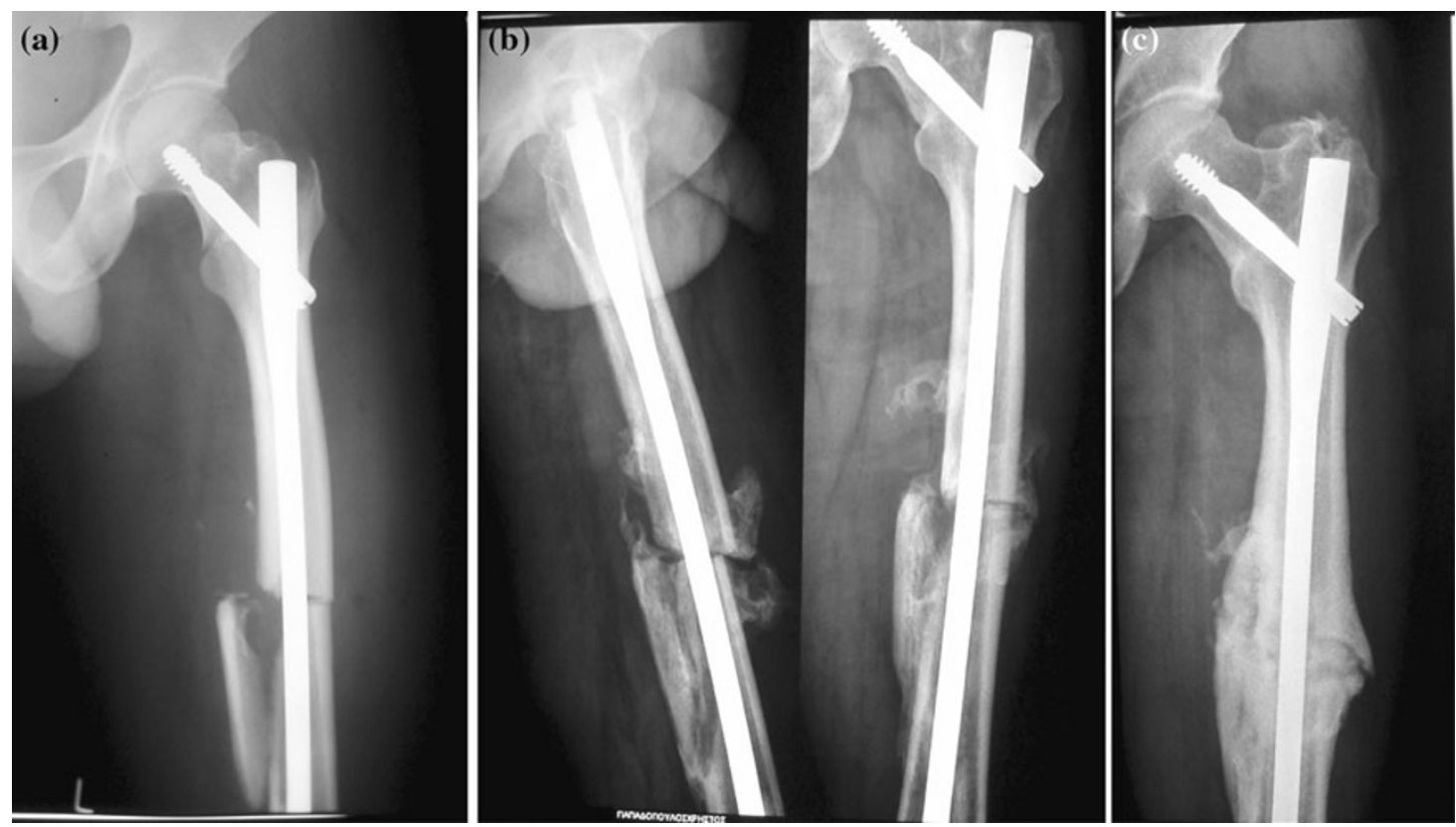

Fig. 3 Radiographs showing ipsilateral neck and shaft fracture (patient No 4): a originally treated with intra-medullary nailing, b hypertrophic non-union occurring 5 months post-operatively and $\mathbf{c}$ satisfactory union achieved after exchange nailing and bone grafting

fixation methods, Tsai et al. [19] found no significant difference in the amount of blood loss, duration of surgery, complications, or clinical results but identified an 11-fold complication rate in the group of antegrade nailing with cannulated screw fixation of the neck fracture compared with the DHS with LCDCP group. In a systematic review of the literature, Bhandari [5] found that the use of separate femoral neck and shaft implants resulted in fewer reoperations than using a single-implant method. However, the absence of prospective randomized trials and the heterogeneity of data available from published case series continue to limit our ability to draw definite conclusions on this issue.

The use of one or two femoral neck screws for the stabilization of femoral neck fractures during reconstruction nailing is debated also. In a prospective comparative study, Vidyathara and Rao [20] found that patients treated with a cephalomedullary nail with two, rather than one, femoral neck screws showed slightly, yet not significantly, better results in terms of functional scores, neck fracture displacement and complication rates. In a recent biomechanical study, Wu and Tai [24] found that supplementary stabilization of the femoral neck fracture with one cannulated screw in front of the reconstruction nail offered better results in terms of load bearing and fracture displacement. In a review of complicated cases, Watson and Moed [21] stressed the poor sliding characteristics of the proximal interlocking screws in second-generation reconstruction nails were accountable for, in their opinion, the varus neck non-unions that occurred in their series. They also suggested that supplementary use of cancellous screws in the femoral neck does not improve neck fracture fixation significantly. Although most of our patients had a single proximal screw, no cases of displacement or malalignment of the neck fracture were met. We conclude that, provided optimal reduction and adequate compression are achieved intra-operatively, a single proximal screw may suffice for the fixation of the neck fracture.

Complications of surgical management include avascular necrosis of the femoral head (AVN), non-union or delayed union of fracture, malunion, fat or pulmonary embolism and infection. The prevalence of AVN ranges between 3 and $4 \%$ in most reported series. Non-union of the femoral neck fracture occurs in approximately 5\% [2]. In this series, there were no cases of AVN. This may have resulted from early surgical intervention and the good quality of fracture reduction.

Femoral shaft non-union or delayed union, in comparison, is more likely. In their series of reconstruction nailing for ipsilateral hip and femoral shaft fractures, Vidyadhara and Rao reported delayed union of the shaft fracture in 22 out of 43 patients. Additional surgery was performed in 6 cases [20]. Similarly, Jain et al. reported a $20 \%$ incidence of femoral shaft non-union using reconstruction nailing [11]. In our patient group, the two implant-related complications both involved the shaft fracture (18\%). This is consistent with the findings by Alho [1] who suggested that, whilst AVN represents the most devastating complication in this setting, it is the union of the shaft fracture which predominantly determines the overall outcome of 
surgical treatment [2]. However, complications involving the femoral shaft are thought to be easier to treat compared with neck fractures $[4,21,23]$. In both our patients, a single additional operation led to satisfactory union.

Limitations of our study include the number of patients examined, its retrospective nature and that no comparison was made between different fixation techniques. However, our results contribute to the limited evidence so far reported on the efficacy of the reconstruction nailing technique in providing stable fixation of these fractures. Here, reconstruction nailing produced, in the mid-term, acceptable clinical and functional results. The implantrelated complications that occurred were successfully treated with a single operative procedure. Further investigation should aim at prospectively comparing all currently available surgical options which, given the rarity of this specific injury, could only be performed on the basis of large multicenter trials.

Open Access This article is distributed under the terms of the Creative Commons Attribution License which permits any use, distribution and reproduction in any medium, provided the original author(s) and source are credited.

\section{References}

1. Alho A (1996) Concurrent ipsilateral fractures of the hip and femoral shaft: a meta-analysis of 659 cases. Acta Orthop Scand 67(1):19-28

2. Alho A (1999) Ipsilateral femoral neck and shaft fractures. J Am Acad Orthop Surg 7(1):76-78

3. Bedi A, Karunakar MA, Caron T, Sanders RW, Haidukewych GJ (2009) Accuracy of reduction of ipsilateral femoral neck and shaft fractures-an analysis of various internal fixation strategies. J Orthop Trauma 23(4):249-253

4. Bennett FS, Zinar DM, Kilgus DJ (1993) Ipsilateral hip and femoral shaft fractures. Clin Orthop Relat Res 296:168-177

5. Bhandari M (2003) Ipsilateral femoral neck and shaft fractures. J Orthop Trauma 17(2):138-140

6. Bose WJ, Corces A, Anderson LD (1992) A preliminary experience with the Russell-Taylor reconstruction nail for complex femoral fractures. J Trauma 32(1):71-76

7. Haidukewych GJ, Rothwell WS, Jacofsky DJ, Torchia ME, Berry DJ (2004) Operative treatment of femoral neck fractures in patients between the ages of fifteen and fifty years. J Bone Joint Surg Am 86(8):1711-1716

8. Heckman JD, Ryaby JP, McCabe J, Frey JJ, Kilcoyne RF (1994) Acceleration of tibial fracture-healing by non-invasive, lowintensity pulsed ultrasound. J Bone Joint Surg Am 76(1):26-34
9. Heiney JP, Leeson MC, Vrabec GA (2009) Delayed diagnosis of an ipsilateral femoral neck fracture with an associated femoral shaft fracture in light of a negative computed tomography scan. J Trauma 67(4):E129-E131

10. Hossam ElShafie M, Adel Morsey H, Emad Eid Y (2001) Ipsilateral fracture of the femoral neck and shaft, treatment by reconstruction interlocking nail. Arch Orthop Trauma Surg 121(1-2):71-74

11. Jain P, Maini L, Mishra P, Upadhyay A, Agarwal A (2004) Cephalomedullary interlocked nail for ipsilateral hip and femoral shaft fractures. Injury 35(10):1031-1038

12. Oh CW, Oh JK, Park BC, Jeon IH, Kyung HS, Kim SY, Park IH, Sohn OJ, Min WK (2006) Retrograde nailing with subsequent screw fixation for ipsilateral femoral shaft and neck fractures. Arch Orthop Trauma Surg 126(7):448-453

13. Peljovich AE, Patterson BM (1998) Ipsilateral femoral neck and shaft fractures. J Am Acad Orthop Surg 6(2):106-113

14. Plancher KD, Donshik JD (1997) Femoral neck and ipsilateral neck and shaft fractures in the young adult. Orthop Clin North Am 28(3):447-459

15. Randelli P, Landi S, Fanton F, Hoover GK, Morandi M (1999) Treatment of ipsilateral femoral neck and shaft fractures with the Russell-Taylor reconstructive nail. Orthopedics 22(7):673-676

16. Swiontkowski MF (1987) Ipsilateral femoral shaft and hip fractures. Orthop Clin North Am 18(1):73-84

17. Swiontkowski MF, Hansen ST Jr, Kellam J (1984) Ipsilateral fractures of the femoral neck and shaft. A treatment protocol. J Bone Joint Surg Am 66(2):260-268

18. Tornetta P 3rd, Kain MS, Creevy WR (2007) Diagnosis of femoral neck fractures in patients with a femoral shaft fracture. Improvement with a standard protocol. J Bone Joint Surg Am 89(1):39-43

19. Tsai CH, Hsu HC, Fong YC, Lin CJ, Chen YH, Hsu CJ (2009) Treatment for ipsilateral fractures of femoral neck and shaft. Injury 40(7):778-782

20. Vidyadhara S, Rao SK (2009) Cephalomedullary nails in the management of ipsilateral neck and shaft fractures of the femurone or two femoral neck screws? Injury 40(3):296-303

21. Watson JT, Moed BR (2002) Ipsilateral femoral neck and shaft fractures: complications and their treatment. Clin Orthop Relat Res 399:78-86

22. Winquist RA, Hansen ST Jr (1980) Comminuted fractures of the femoral shaft treated by intramedullary nailing. Orthop Clin North Am 11(3):633-648

23. Wiss DA, Sima W, Brien WW (1992) Ipsilateral fractures of the femoral neck and shaft. J Orthop Trauma 6(2):159-166

24. Wu CC, Tai CL (2009) Reconstruction interlocking nails for ipsilateral femoral neck and shaft fractures: biomechanical analysis of effect of supplementary cannulated screw on intracapsular femoral neck fracture. Clin Biomech (Bristol, Avon) 24(8): 642-647 